\title{
Newtonian and Non-Newtonian Pulsatile Flows through an Artery with Stenosis
}

\author{
P. Haldera, A. Husaina, M. Zunaid ${ }^{\mathrm{b}}$ and A. Samad ${ }^{*}, \mathrm{c}$ \\ a Department of Mechanical and Industrial Engineering, Sultan Qaboos University, Muscat, PC-123, Oman. \\ ${ }^{b}$ Department of Mechanical Engineering, Delhi Technological University, Delhi-110042, India. \\ c Department of Ocean Engineering, Indian Institute of Technology Madras, Chennai-600036, India.
}

Received 14 March 2017; Accepted 16 October 2017

\begin{abstract}
The current study presents three-dimensional modeling and analysis of blood flow through artery stenosis under several variants of pulsatile flow to mimic the atherosclerosis artery disease. The study considered Newtonian and non-Newtonian blood flow models and solved the threedimensional laminar and unsteady Navier-Stokes (NS) equations for different inlet velocity profiles. The Carreau Yasuda model was used for hemodynamics modeling of fluid viscosity. A comparative analysis was carried out for Newtonian and non-Newtonian fluid models under several simple pulsatile, equivalent pulsatile and physiological velocity profiles. The non-Newtonian fluid exhibited a higher centerline velocity. Wall shear stresses, shear strain rates, velocity fields, and vortex distribution for Newtonian and non-Newtonian fluid flows show significant differences. A higher wall shear was noticed near the stenosis. The vortex formed near the stenosis shifts to upstream and downstream of the stenosis with the change of velocity pulse. The velocity profile deflates more for non-Newtonian flows than the Newtonian flows.
\end{abstract}

Keywords: Non-newtonian fluid; Blood flows; Artery stenosis; Physiological flows; Pulsatile flows.

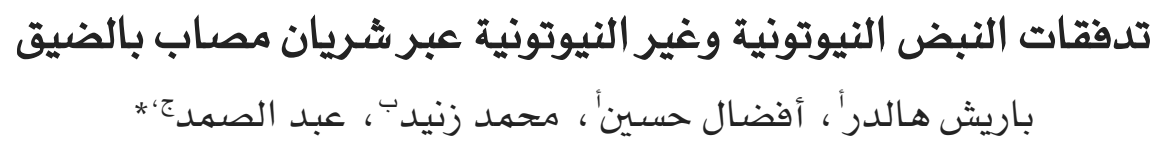

الملخص : قامت الدراسة الحالية بتقديم نمذجة ثلاثية الأبعاد وتحليل لتدفق الدم عبرشريان مصاب بضيق وذلك تحت عدة تدفقات نبض متفيرة و ذلك لمحاكاة مرض تصلب الشرايين. وأخذت الدراسة يخٌ الاعتبار نماذج تدفق الدم النيوتونية وغير النيوتونية وحلت الرقائق ثلاثية الأبعاد و معادلة نافيير ستوكس (نس) و معادلات الاشكال المختلفة للسرعة عند المدخل.وقد تم استخدام نموذج كاريو ياسودا للزوجة السوائل من أجل نمذجة ديناميكا الدم .و تم اجراء تحليل مقارن لنهاذج السوائل النيوتونية وغير النيوتونية تحت عدة نبضات بسيطة مضاهية لاشكال سرعة النبض الفسيولوجيي. وقد أظهر السـائل غير النيوتوني سرعة أعلى وِخ خط الوسط. كما تبين وجود اختلافات كبيرة ِّ إجهاد القص، ومعدلات القص و مسـارات السرعة وتوزيع كتلة الدوامـة على السوائل النيوتونية وغير النيوتونية. و قد لوحظ وجود ارتفاع عْ جدار القص بالقرب من منطقة الضيق و تتقل كتلة الدوامة المتكونة بالقرب من منطقة الضيق من المدخل الى

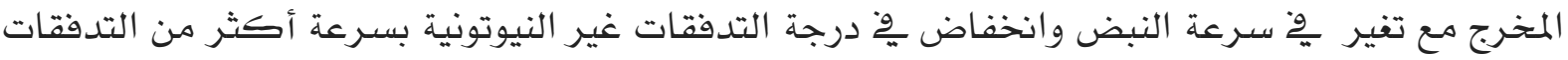
النيوتونية. الكلمات المفتاحية : التدفقات النابضة ، السوائل غير النيوتونية تدفقات الدم. ضيق الشريان. التدفقات

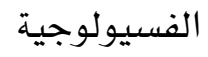

* Corresponding author's e-mail: samad@iitm.ac.in 


\section{Introduction}

The primary function of the cardiovascular system is to transport nutrients and oxygen to different body parts through blood circulation in arteries. Atherosclerosis or artery stenosis is an artery disease that causes artery constriction due to the decomposition of smooth muscle cells and to the presence of lipids in the artery that form plaque in the artery wall. These plaques grow gradually with time and block the flow path. The hemodynamic effects of artery stenosis are far reaching beyond the reduced artery section, and dictates normal and shear stress distribution and low-pressure zones near the constricted region. The atherosclerosis plaque forms in the high shear region; therefore, it necessitates the investigation of the dynamic effect of the constriction on blood flow before and after the formation of atherosclerotic lesions. The cardiovascular system of bifurcated tubes supplies nutrition to different organs of the body with the help of heart pump. By increasing or decreasing their cross-sectional area, the arteries that are far from the inert tubes adapt to changing pressure and blood flow conditions to accommodate the hemodynamic demands. It is important to study the blood flow in arteries with physiological conditions, where the blood flow demand changes under disease conditions.

Several numerical and experimental studies have been reported to comprehend the behavior of blood flow through arteries. Mcdonald (1955) studied pressure oscillations generated flow through the femoral artery of a dog. Young and Tsai (1973) experimentally investigated the effect of axisymmetric and non-symmetric plastic models with different area ratios and constriction lengths. The test results showed that geometry has a strong influence on the flow characteristics, which includes size and shape of the stenosis. Deshpande et al. (1976) carried out computational analysis for steady blood flow through an artery with axisymmetric-contoured constriction and presented comparison with experimental data. The reported results were well-matched with the existing experimental results (Young and Tsai 1973). The flow field in human vasculature is relatively complex, and the carotid sinus exerts a high impact on the hemodynamics at the bifurcation (Bharadvaj et et al. 1982a; Bharadvaj et al. 1982b). MazHer and Ekaterinaris (1988) modled three-dimensional blood flows as Newtonian fluid flows incorporating secondary and separated flows under steady conditions.

Most of the above studies are based on steady flow analysis whereas the heart delivers a pulsating flow, which is unsteady in nature. Later, several authors investigated pulsatile Newtonian and non-Newtonian flows in artery bifurcation. Perktold et al. (1991) reported numerical results for Newtonian and nonNewtonian fluid flow through a carotid artery bifurcation model. The result showed that the fluid variables in the sinus get affected by the angle between the internal carotid axis and the common carotid axis. Perktold and Rappitsch (1995) analyzed the effect of the distensible artery wall on the local flow field and determined the mechanical stresses on the artery wall for a non-Newtonian inelastic fluid. Further, Ku et al. (1985) measured pulsatile flows in a scaled model of the human carotid bifurcation by a laser doppler velocimetry. Tu et al. (1992) computationally investigated flow through a stenosis in a rigid circular tube based on a two-dimensional Galerkin finite element method. The paper reported the efffects of constriction severity, stricture length and Reynolds number on flow vortex and pressure distribution. The sharp peaks of WSS (wall shear stress) were present near the throat of the stenosis and presusre recovered partly at downstream of the stenosis. Deplano and Siouffi (1999) experimentally and numerically investigated different types of constriction shapes and the two results were in good agreement. Zendehbudi and Moayeri (1999) indicated that the behaviour of the pulsatile flow is completely different from physological flow. Razavi et al (2011) investigated the flow dynamics related to a common carotid artery with symmetric stenosis varying severity 30$60 \%$ under Newtonian and non-Newtonian viscosity models.

Furthermore, Chakravarty and Mandal (1994) developed a mathematical model for blood flow in a stenosed arterial segment using a pulsatile pressure gradient method, and showed that stenosis affects the WSS distribution. Tu and Deville (1996) used Herschel-Bulkley, Bingham, and power-law models of non-Newtonian fluids to analyze the flow through arterial stenosis and discussed pressure distribution, flow pattern, and WSS distribution. Liao et al. (2004) investigated a bellshaped stenosis for three different pulsatile flows and concluded that the fluctuations of flow parameters affect the flow field for the 
physiological flow. Srivastava and Rastogi (2010) investigated an axially nonsymmetrical stenosis blood flow through a narrow catheterized artery. The results showed that the impedance enhances with the catheter, the hematocrit and the stenosis size decreases with the shape of parameter. Nadeem et al. (2011) investigated tapered artery with stenosis using power law fluid model and examined velocity, impedance (flow resistance), WSS and stress at the stenosis throat. Ellahi et al. (2014) studied the mathematical models of Jeffrey fluid with nanoparticles in the tapered atherosclerotic arteries. The authors solved mathematical model for mild stenosis. Mekheimer and El Kot (2015) investigated the axisymmetric flow of blood through curved coaxial tubes. A significant difference was noticed between the flows through curvature and non-curvature annulus flows through catheterized stenosed artery for the axial velocities, the stream function, the impedance, pressure drop and the WSS distribution.

Due to the complex nature of fluid and flow modeleing, most of the above studies presented conservative two-dimesional analysis. Further, research was carried out to investigate threedimensional flow dynamics of artery blood flows. Ang and Mazumdar (1997) developed a mathematical model for blood flow through an asymmetric arterial stenosis. A higher shear stress was noticed on the wall with stenosis as compared to the other side with no protuberance for the flow with Reynolds number ranging from 100 to 1000 and for four degrees of stenosis severity. Long et al. (2001) investigated pulsatile flow through asymmetrical and axisymmetrical stenosis models with $25 \%, 50 \%$ and $75 \%$ constrictions and reported that the stenosis influence length is shorter for asymmetrical models. Hye and Paul (2015) studied the spiral and non-spiral blood flows with axisymmetric arterial stenosis by using two-equation standard $\mathrm{k}-\omega$ model and Large Eddy Simulation (LES). The results showed that the spiral flow disturbs the turbulence kinetic energy in the post stenosis region. Chiastra et al. (2017) studied the coronary bifurcation anatomical features on both near-wall and intravascular flow features for a healthy and diseased artery. The bifurcation angle effects are small on hemodynamics in both cases of artery with or without stenosis.

A lot of research has been carried out to conservative modeling of artery bifurcation and stenosis, there is a lack of deterministic modeling of flow through diseased artery with realistic fluid model, flow conditions, physiological pulse and elastic artery wall. The present research is part of comprehensive numerical analysis carried out to investigate flow through stenosed artery with realistic flow modeling and physiological conditions. In this study, hemodynamics of Newtonian and nonNewtonian fluids in an artery with a symmetric stenosis for simple and equivalent pulsatile and physiological blood flows are studied through three-dimensional numerical modelling assuming rigid artery walls. The comparative analysis is presented for Newtonian and nonNewtonian flows under variants of pulsatile flows. The several flow characteristics have been investigated for velocity, vortex, wall shear stress and shear strain rates to demonstrate blood flow dynamics at different intervals of flow cycle under different flow conditions.

\section{Mathematical Model and Numeri- cal Method}

An artery with a symmetrical stenosis was taken for numerical analyses as shown in Fig. 1. The cylindrical coordinate system is considered with variable coordinates $(r, z)$. The stenosis profile can be defined mathematically as follows (Liao et al. 2004):

$$
\begin{gathered}
\frac{r}{r_{0}}=1-\frac{\delta}{2 r_{0}}\left(1+\cos \frac{\pi z}{z_{0}}\right) \\
-z_{0} \leq z \leq z_{0}
\end{gathered}
$$

where, $r$ is the artery radius with the function of $z$ in the stenosis region, $L$ is the length of the artery and $r_{0}$ is the radius of the artery. In this case, $\delta=0.5$ and $z_{0}=1.0$ (Liao et al. 2004).

Figure 1 represents the computational domain for numerical analysis. The CAD model was setup in ANSYS design modular. The mesh generation tool ANSYS Mesh was employed to build the structured mesh for the computational domain. The number of mesh elements are increased gradually from 141102 to 840164 to check the grid dependence of the solution. The results showed that there is no a significant difference in velocity profiles for the change of mesh elements from 325017 to 840164 . Therefore, a grid with about 600000 elements is considered as optimum one (Fig. 2). No slip boundary condition was applied for the artery 
wall whereas time dependent velocity was assigned at the inlet boundary. The outlet was assigned as an opening type boundary condition with pressure outlet. The static pressure at the outlet was kept as zero atm, while the reference pressure was one atm.

The governing equations for the model are as follows:

The continuity equation yields

$$
\frac{\partial \rho}{\partial t}+\nabla \cdot(\rho U)=0
$$

And momentum equations

$\frac{\partial(\rho U)}{\partial t}+\nabla \cdot(\rho U \otimes U)=-\nabla p+\nabla \cdot \tau$

Where the stress tensor, $\tau$ is related to the strain rate by

$\tau=\mu\left(\nabla U+(\nabla U)^{T}-\frac{2}{3} \delta \nabla . U\right)$
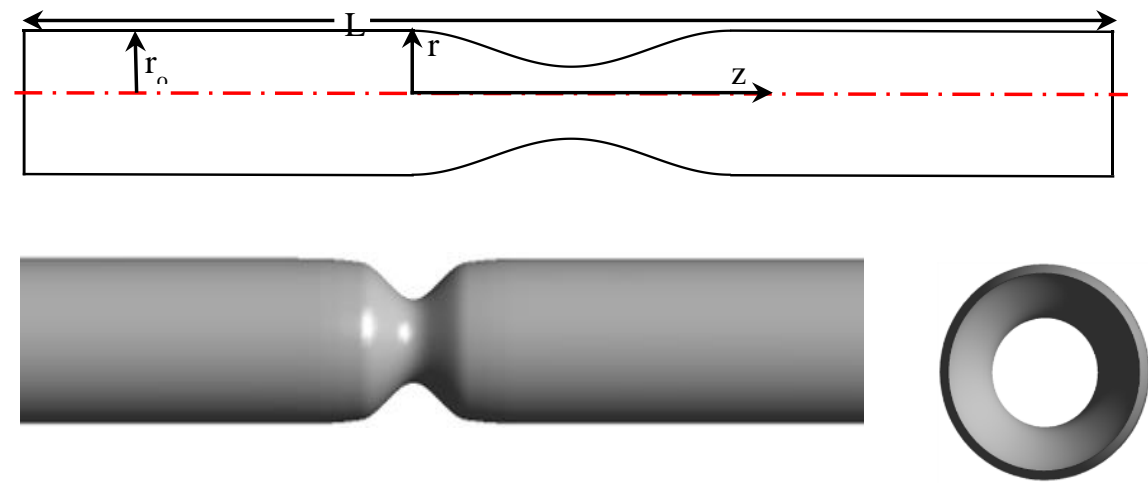

(a)

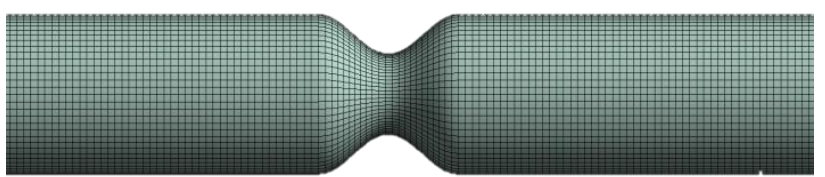

(b)

Figure 1. (a) Schematic diagram and computational domain, and (b) grid distribution.

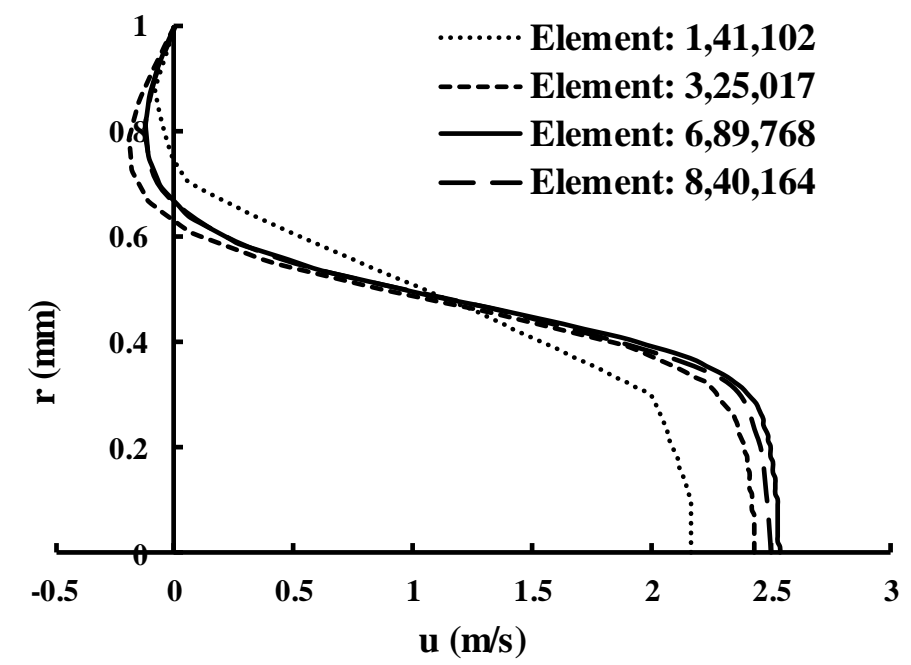

Figure 2. Grid Test. 
In the above equations, $\rho, U, p$ and $t$ represent density, velocity, pressure and time, respectively. A finite volume based solver ANSYS-CFX (Ansys CFX 2010) was used to solve the unsteady three-dimensional incompressible Navier-Stokes (NS) equations to model the flow in artery. It is a coupled pressure based solver which solves velocity and pressure equations as a single system (Ansys CFX 2010) and uses SIMPLEC (Semi-Implicit Method for Pressure Linked Equations-Consistent) algorithm for pressure correction (Van Doormaal and Raithby 1984). The equations were discretized by the cell-centered finite volume approach. High resolution and first order discretization schemes were implemented in the computations. The discretized system of equations was solved using a multigrid accelerated ILU (Incomplete lower-Upper) factorization procedure at any given time step and the solution was marched in time through fully implicit procedure. For each time step, the spatial field was solved through several iterations to reach to the conversion criteria. The required root mean square (RMS) convergence criteria for the momentum and continuity equations were $1 \times 10^{-9}$.

Blood density depends on age and sex and it is varied within the range $1030-1070 \mathrm{~kg} / \mathrm{m}^{3}$ (Jozwik and Obidowski 2008). For conservative analysis, the density of the blood was assumed constant as $1058 \mathrm{~kg} / \mathrm{m}^{3}$ for both Newtonian and non-Newtonian fluids. The flow analysis was done for both Newtonian and non-Newtonian fluids. The dynamic viscosity for the Newtonian fluid was kept constant (0.004 Pa.s) whereas for the non-Newtonian fluid the Carreau-Yasuda model was implemented which is expressed as:

$$
\mu=\mu_{\infty}+\frac{\left(\mu_{o}-\mu_{\infty}\right)}{\left(1+(\lambda \dot{\gamma})^{a}\right)^{\frac{1-n}{a}}}
$$

Here, low shear viscosity, $\mu_{0}=0.056$ Pa.s; high shear viscosity, $\mu_{\infty}=0.00345$ Pa.s; Time Constant, $\lambda=3.3313 \mathrm{~s}$; Power Law Index, $n=$ 0.3568; Yasuda Exponent, $a=2$ (Ahsaas and Tiwari 2016a). The Carreau-Yasuda model is continuous for a wide range of flow shear rates $(\dot{\gamma} \geq 0)$. For high shear thinning the fluid viscosity approaches to $\mu_{\infty}$, whereas for low shear thinning it approaches to $\mu_{0}$ which mimics well blood flow modeling (Razavi et al. 2011; Ahsaas and Tiwari 2016; Gijsen et al. 1999).

\section{Results and Discussion}

In this work, three common velocity profiles, i.e. simple pulsatile, equivalent pulsatile and physiological flows, were used to describe the arterial flows (Mcdonald 1955). The cyclic variations of the velocities are given in Fig. 3. The simple pulsatile and the equivalent pulsatile flows are expressed as:

Simple pulsatile flow:

$U(t)=1+\sin \left(t^{*}\right)$

Equivalent pulsatile flow:

$U=1-\alpha\left(1+\cos \left(t^{*}+\phi\right)\right)$

where $t^{*}$ is the non-dimensional time $(2 \pi t / T), \mathrm{T}$ is the time period, $a$ is the amplitude and $\phi$ is the phase angle. The variation of velocity for simple pulsatile flow is symmetric about zero velocity over the time, whereas equivalent velocity profile has positive velocity for the larger part of the cycle. The numerical model was validated and the velocity profiles were compared with the ones reported in literature by Deshpande $e t$ al. (1976) and Liao et al. (2004) for fully developed laminar flow through artery stenosis at two locations $(z=0.001$ and $z=10.26)$. The velocity profiles predicted by present model are in good agreement with the reference profiles except the slight deviation near the walls which appears due to the three-dimensional nature of the flow considered in the present study as compared to the two-dimensional behavior reported in the reference studies (Deshpande $e t$ al. 1976; Liao et al. 2004) (Fig. 4).

The steady state hat fully developed flow field is generated through simulations and the results were compared with Newtonian and non-Newtonian fluids. Fig. 5 illustrates velocity and streamline distribution at the mid plane of the artery. The higher velocity region extends longer downstream to stenosis along with greater vertical regions near the walls for the Newtonian fluid than the non-Newtonian fluid. The non-Newtonian fluid gives a deflated velocity profile and high velocity gradients are concentrated near the walls; whereas, the Newtonian fluid exhibits a high velocity core downstream to the stenosis due to shear thinning. The separation regime spreads downstream of the stenosis much longer for the Newtonian fluid than the non-Newtonian fluid. 


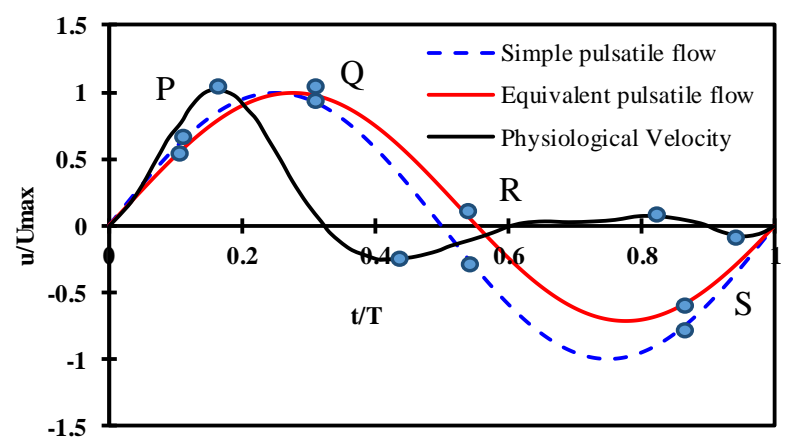

Figure 3. Velocity profile with time for simple pulsatile, equivalent pulsatile and physiological flows.

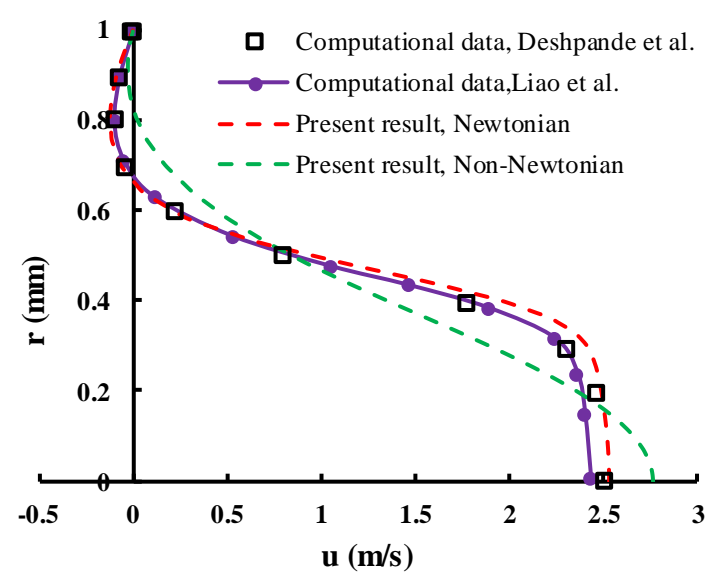

(a) $\mathrm{Z}=0.001$

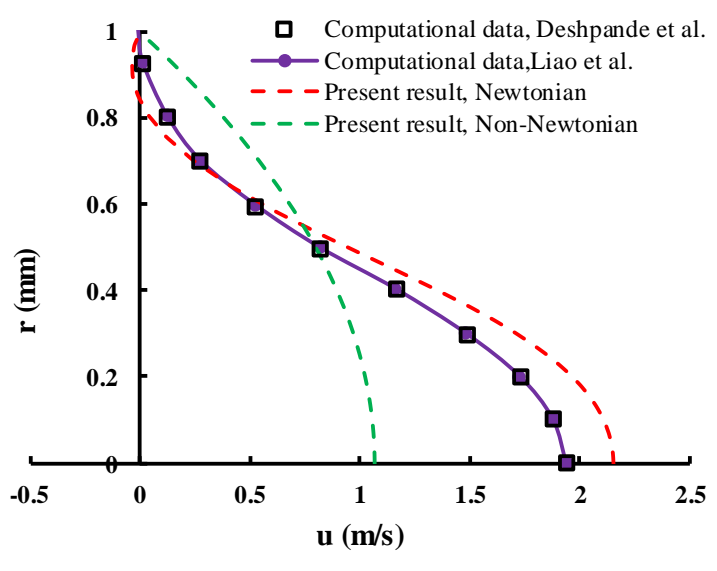

(b) $\mathrm{Z}=10.26$

Figure 4. Validation of numerical model with existing computational data at $\operatorname{Re}=200$ (Deshpande et al. 1976; Liao et al. 2004).

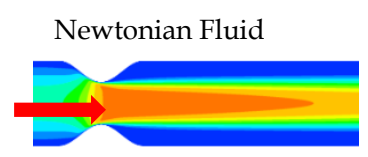

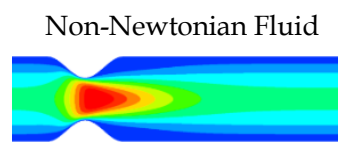

Velocity
Newtonian Fluid

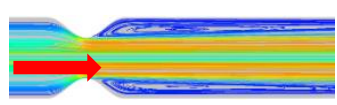

$\left[\mathrm{m} s^{\wedge}-1\right]$

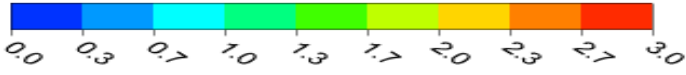

Non-Newtonian Fluid

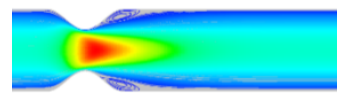

Figure 5. Velocity distribution and streamines at the mid-section tor Newtonian and Non-Newtonian flow.

Both fluids produce a higher shear strain rate in the wall near the artery stenosis, but the strain rate is higher at the proximal upstream and lower at the proximal downstream of the stenosis (Fig. 6). The non-Newtonian fluid shows high wall shear stresses appeared in the larger part of the artery wall because of the higher viscosity, while, the Newtonian fluid shows a higher shear strain rate.

Figure 7 shows velocity distribution for steady state fully developed flow at different streamwise locations, i.e. upstream to stenosis plane at $\mathrm{z}=-0.001 \mathrm{~m}$, middle of the stenosis, $\mathrm{z}=$ $0.0 \mathrm{~m}$, and downstream to stenosis planes, $\mathrm{z}=$ $0.001 \mathrm{~m}$ and $\mathrm{z}=0.01 \mathrm{~m}$. The velocity distribution is almost similar at the upstream of the stenosis for both fluids. A deflated velocity profile for the non-Newtonian fluid appears at the proximal and distal downstream of the stenosis due to shear thinning.

Furthermore, transient flow analysis was carried out for both Newtonian and nonNewtonian fluids for simple pulsatile, equivalent pulsatile and physiological flows and 
flow characteristics at some discrete locations in the artery are compared as shown in Fig. 8. A higher velocity appears at the middle of the stenosis (Point C) and proximal downstream to stenosis (Point D) than the other upstream and downstream locations for the simple pulsatile flow for Newtonian and non-Newtonian fluid due to the constriction near the stenosis. For equivalent pulsatile and physiological flows, the velocity is maximum at and proximal to stenosis during the forward pulse, which is typically similar to simple pulsatile flow, whereas during the backward pulse the location of the maximum velocity $\left(U_{\max }\right)$ is shifted towards upstream side. A significant velocity fluctuation appears at point E over the time due to the growth of flow disturbances (Fig. 8(b) and (c)). The maximum velocity $\left(U_{\max }\right)$ is observed at the middle and proximal downstream of stenosis (Point $C$ and Point D) for all the cases. The $U_{\max }$ appears twice during the cycle for simple pulsatile flow, during the forward pulse for equivalent pulsatile and during the first forward pulse for physiological flows. The minimum velocity $\left(U_{\min }=0\right)$ is observed at all points at different time intervals, i.e. at 3T/4 for simple pulsatile flows, at $0 \mathrm{~T}, \mathrm{~T} / 2$ and $\mathrm{T}$ for equivalent pulsatile and at several time instants for physiological flows as shown in Fig. 8.

The flow field is further analyzed for streamlines, shear strain and shear stress at different time intervals for both Newtonian and non-Newtonian flows with simple pulsatile, equivalent pulsatile and physiological flows. Figure 9 shows streamlines for the simple pulsatile flow at different time intervals for both Newtonian and non-Newtonian fluids at the middle plane of the artery. The flow field development is almost similar to both the fluids because of the similar velocity waveform in the first and second half-cycle. The mean velocity of the flow is higher in the forward half-cycle than the backward one. The asymmetry of the flow appears during the forward half cycle of Newtonian fluid due to the growing recirculation and disturbance in the flow

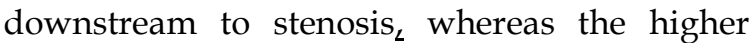
viscosity resulting from the higher shear thinning suppresses the flow disturbances for non-Newtonian fluid (Fig. 9). Though, both fluids produce recirculation downstream of the stenosis, due to lower viscosity, Newtonian fluid produces a larger recirculation regime.

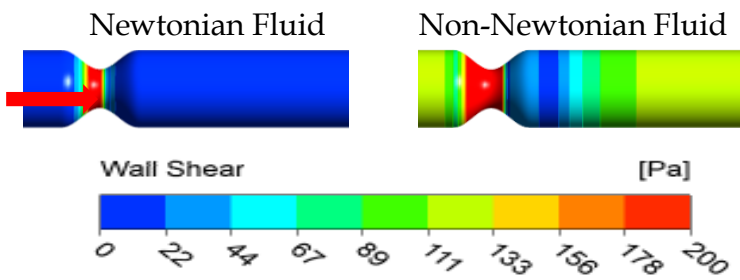

Figure 6. Shear strain rate and shear stress distribution at the wall.

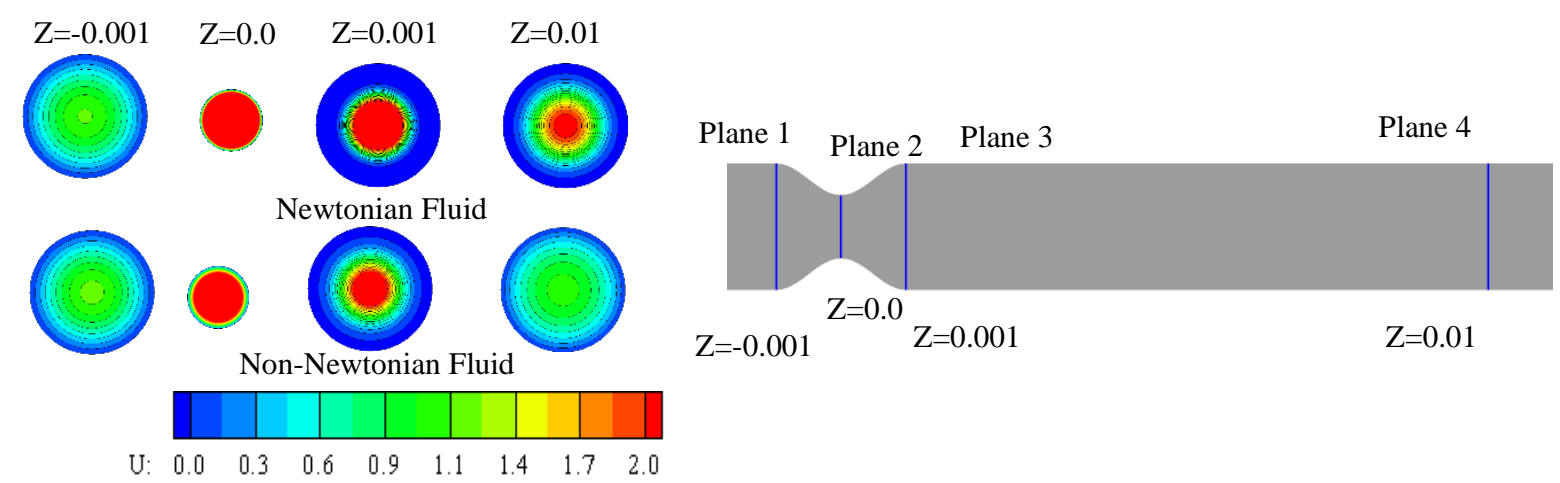

Figure 7. Velocity distribution at several data reduction planes. 


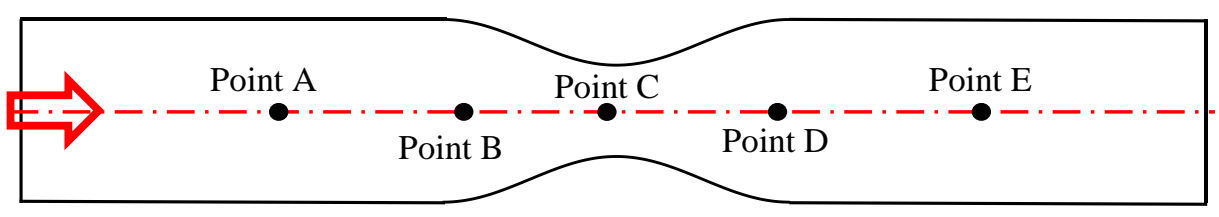

(a)

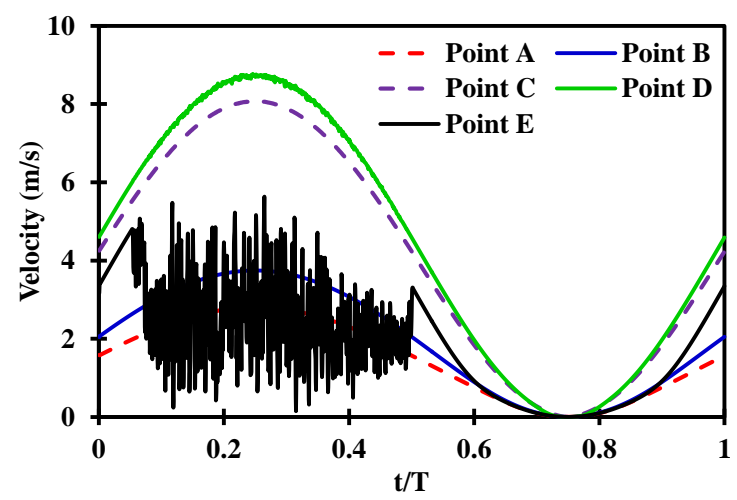

Newtonian fluid

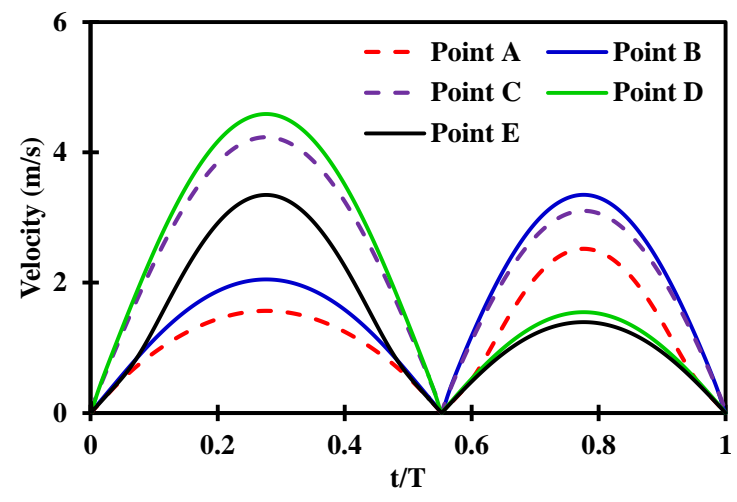

Newtonian fluid

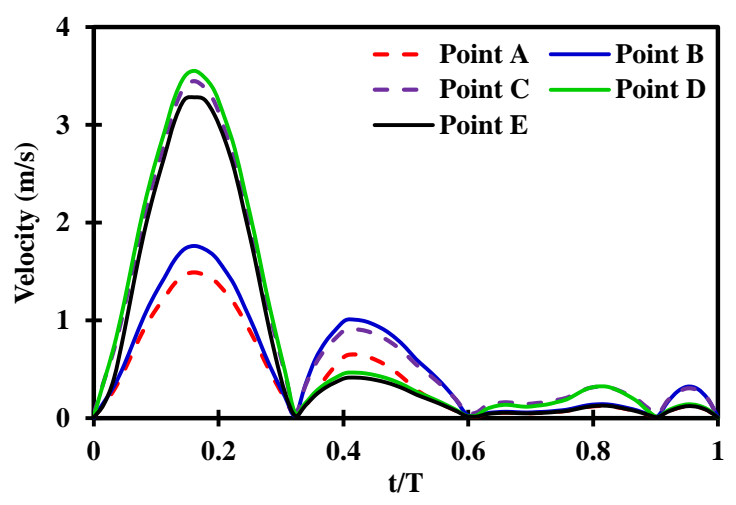

Newtonian fluid

(d)

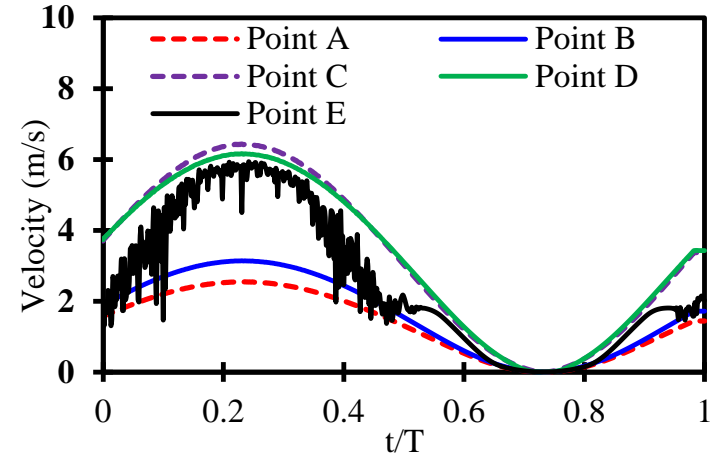

(b)

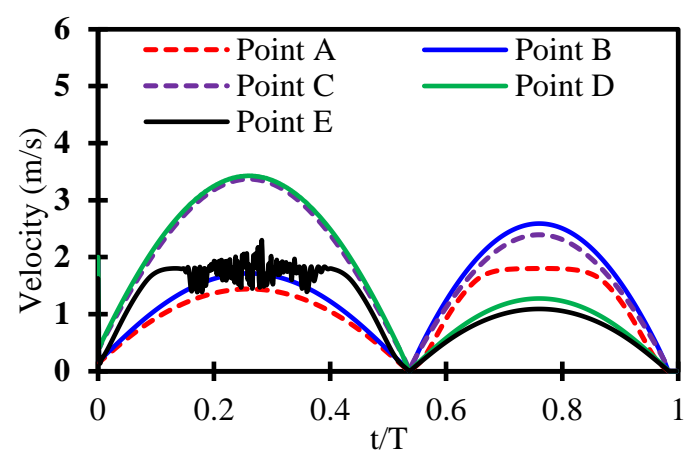

(c)
Non-Newtonian fluid

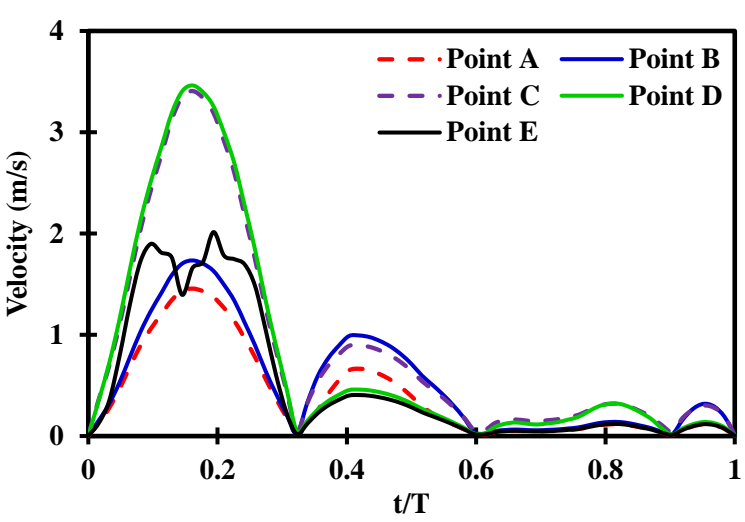

Non-Newtonian fluid

Figure 8. (a) Data reduction point probes, and velocity distribution for (b) simple pulsatile, (c) equivalent pulsatile, and (d) physiological flows, at different locations for Newtonian and non-Newtonian flows. 
Figure 10 shows the wall stress shear (WSS) and shear strain rate distribution at different time intervals for simple pulsatile flows. The induction of WSS facilitates atherosclerotic processes that affect the formation and rupture of atheromatous plaque. For the non-Newtonian fluid having higher viscosity, a higher WSS is produced near the bifurcation region, and the maximum WSS lies between $\mathrm{z}=-0.001 \mathrm{~m}$ to $\mathrm{z}=$ 0.0m (Fig. 10). For Newtonian fluid, a higher shear strain rate is noticed near the throat during the forward cycle.

Figure 11 shows the velocity distribution along the streamwise direction at different locations $(Z=0.001,0.0,0.001$ and 0.01$)$ and at different time intervals. For the Newtonian fluid, the flow parameter changes significantly, and the changes are more dominant at $Z=0.001$ and $Z=0.01$. The symmetry of the flow is broken as the flow past stenosis due to the growing disturbances created by velocity pulse for the Newtonian fluid which necessitates threedimensional modeling of the flow. As for nonNewtonian fluid, these disturbances are suppressed and symmetry is maintained.

The transient effect of the velocity pulse on the artery flow field is analyzed through velocity and pressure distribution along the centerline in the artery at several time intervals for simple pulsatile flow (Fig. 12). The velocity increases gradually along the flow direction up to the middle of the stenosis and sharply near the stenosis due to artery constriction. Furthermore, velocity decreases gradually post stenosis until the end of the artery. The maximum and the minimum velocity in the artery appear at $\mathrm{t}=0.3 \mathrm{~s}$, and $0.7 \mathrm{~s}$, respectively.

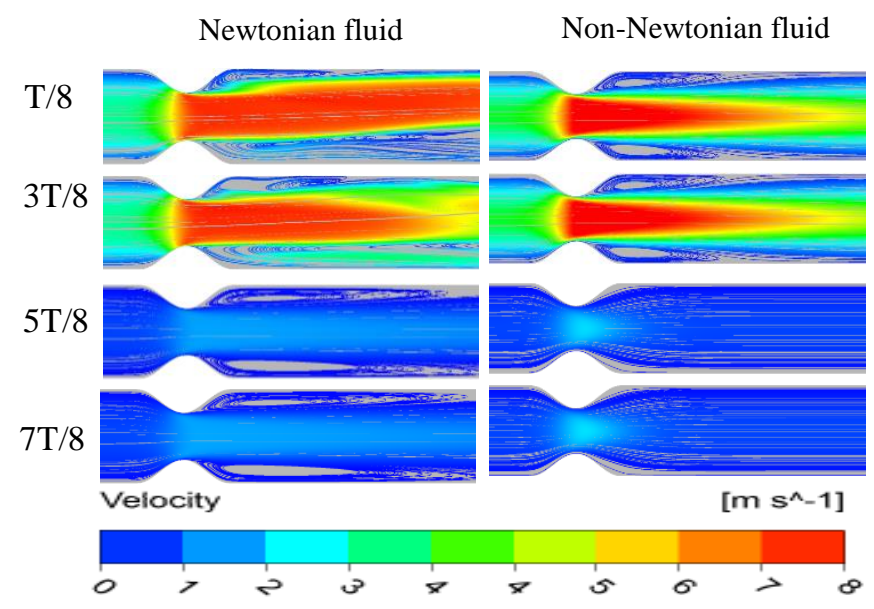

Figure 9. Streamlines at the mid-plane along the flow direction for simple pulsatile flows.

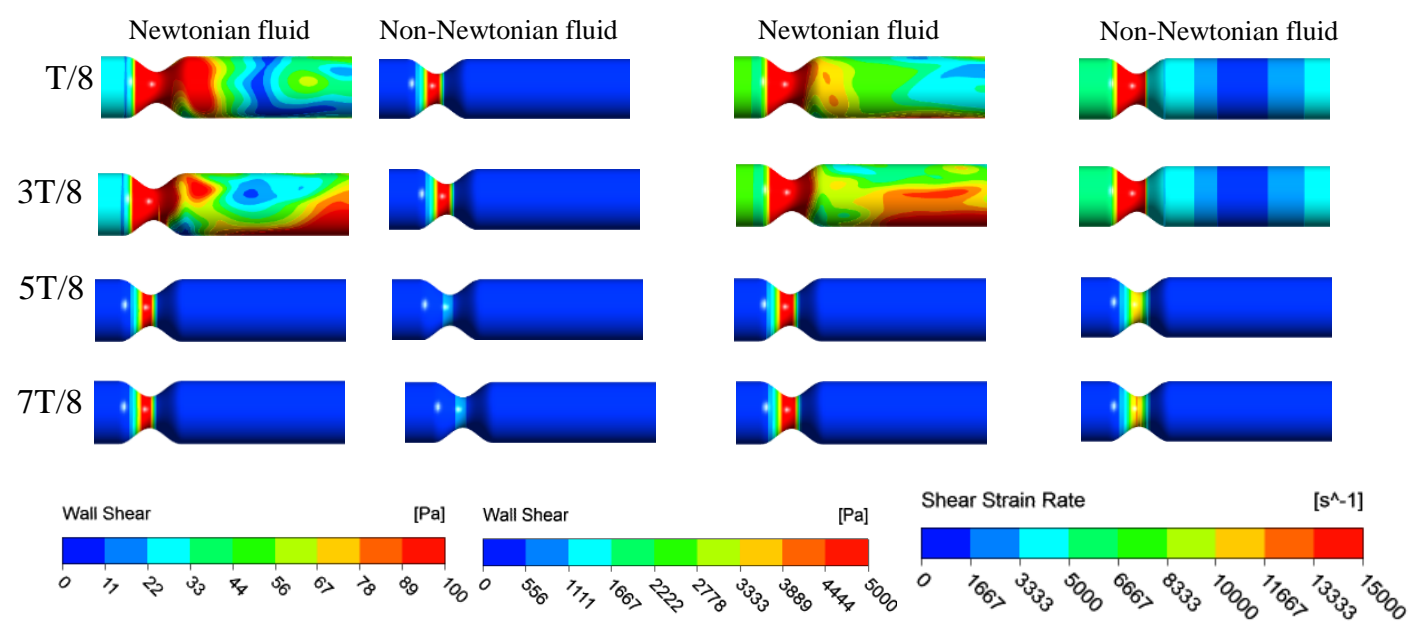

Figure 10. Wall stress shear and shear strain rate distribution at different time for simple pulsatile flows. 


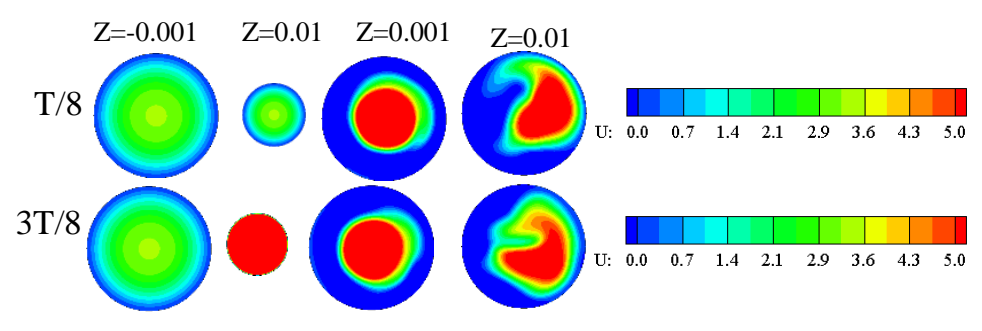

Newtonian fluid
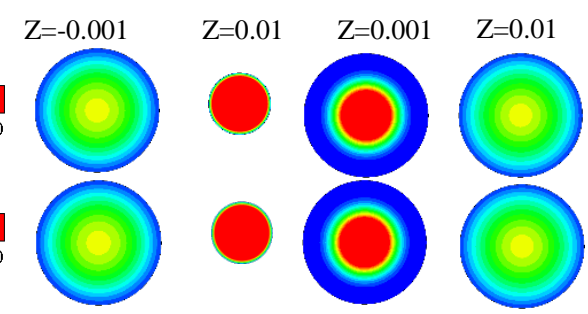

Non-Newtonian fluid

Figure 11. Velocity distribution at different locations in the flow direction for simple pulsatile flows.
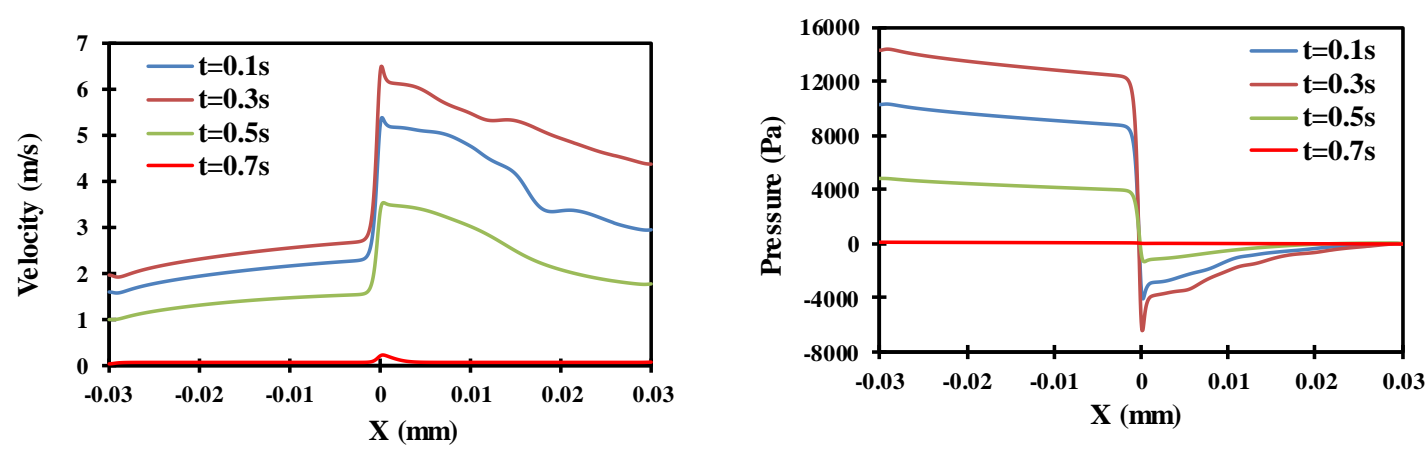

(a)
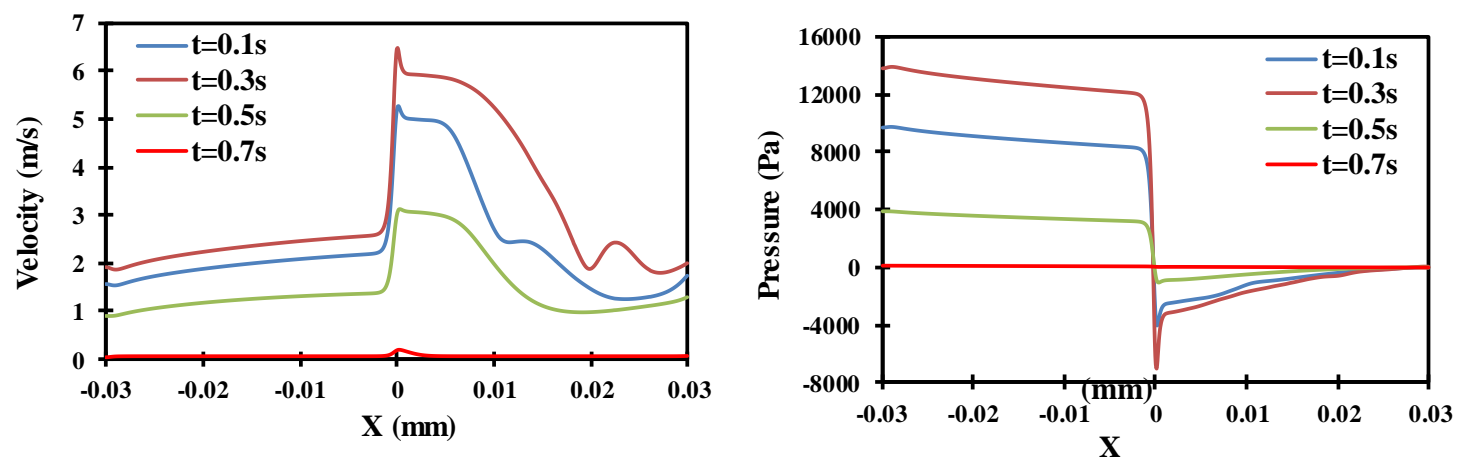

(b)

Figure 12. Velocity and pressure distribution on the central line of the artery for (a) Newtonian and (b) Non-Newtonian pulsatile flows at different time intervals.

The pressure in the artery first decreases gradually until the stenosis center then decreases sharply in the middle of the stenosis, which is then followed by gradual increase until the end of the artery. The minimum pressure appears at $\mathrm{t}=0.3 \mathrm{~s}$ at proximal downstream of the stenosis indicating the regime prone to atherosclerotic processes.

The streamlines at different time instances for the equivalent pulsatile flow are shown in Fig 13. The Newtonian fluid shows a uniform velocity profile in the core of the artery, whereas the non-Newtonian fluid shows a velocity balloon near the stenosis. For the Newtonian fluid, a large vortex covers the central core in both forward and backward pulses. The non-
Newtonian fluid shows a small vortex only at the proximal region to the stenosis, i.e., downstream during the forward cycle and upstream during backward cycle. The nonNewtonian fluid flow produces a higher WSS at all instances, a higher shear strain rate at all locations other than the downstream of the stenosis (Fig. 14). Both fluids give higher WSS near the stenosis. The pressure and velocity distribution along the central line for equivalent pulsatile flow at different time intervals are shown in Fig. 15. At $t=0.3 \mathrm{~s}$, the peak velocity appears at the throat of the stenosis for the forward cycle.

Figure 16 shows streamlines for physiological flows for both fluids at different 
time intervals. The location of the vortex, which is proximal to the stenosis, keeps changing its location as the velocity pulse reverses its direction during the cycle. Both fluids give a larger vortex during the first quarter. The temporal characteristics at the central line in the artery are shown in Fig. 17 at different time intervals. The maximum velocity which is associated with the minimum pressure appears at $\mathrm{t}=0.125 \mathrm{~s}$ for physiological flows, while the peak velocity is occurred at $t=0.3 \mathrm{~s}$ for simple and equivalent pulsatile flows.
The present research reports numerical modelling of blood flows through an artery with a stenosis for hemodynamic characteristics of atherosclerosis. The model was set up for Newtonian and non-Newtonian fluid flows for three commonly used velocity profiles, i.e. simple pulsatile, equivalent pulsatile, and physiological flows. The computational model was validated and results were compared with the results available in the open literature. The Following conclusions can be drawn based on the findings of the present study:

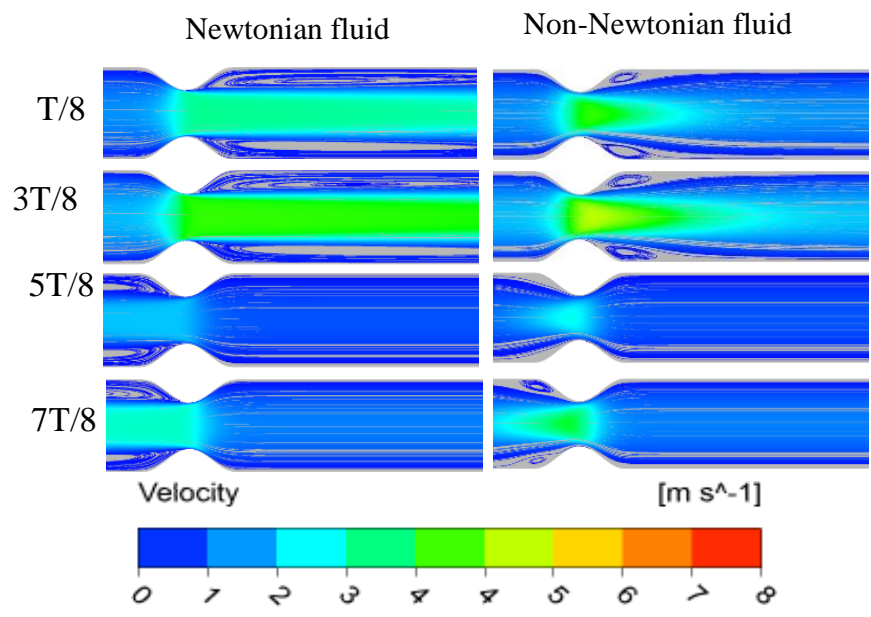

Figure 13. Streamlines at the mid-plane along the flow direction for equivalent pulsatile flows.

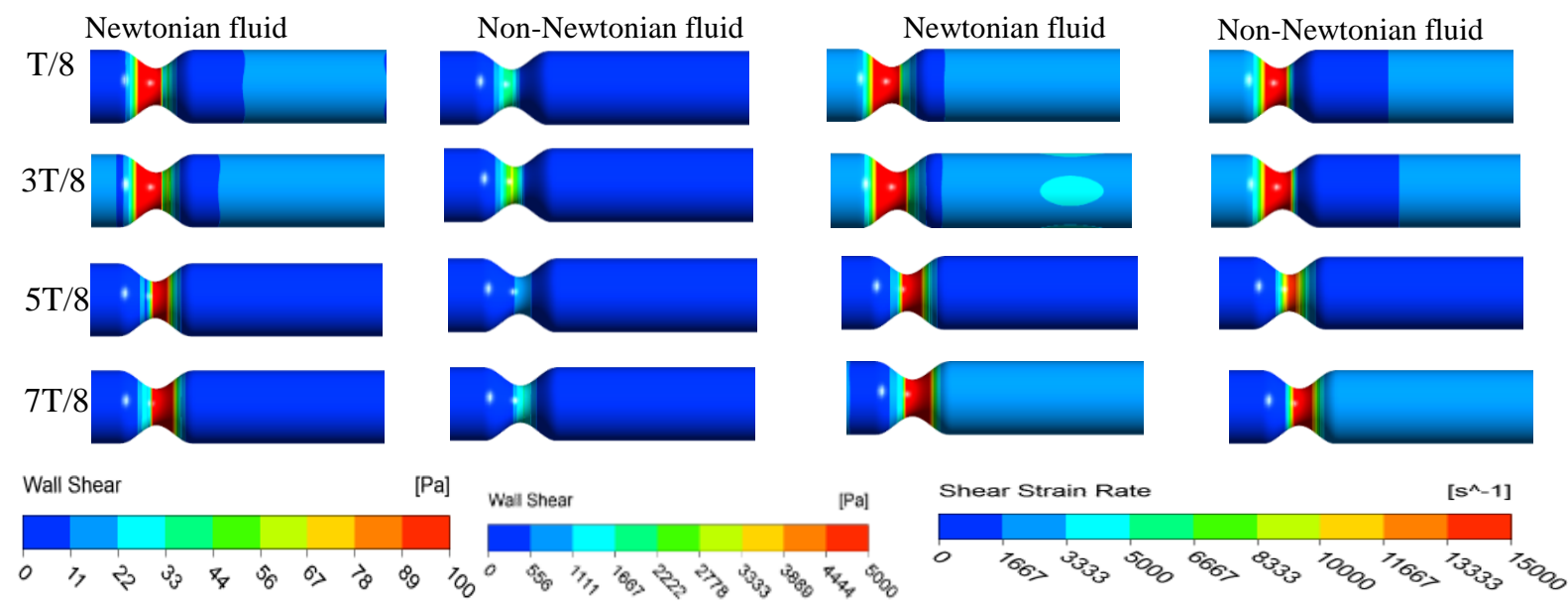

Figure 14. Wall stress shear and shear strain rate distribution at different time for equivalent pulsatile flows. 

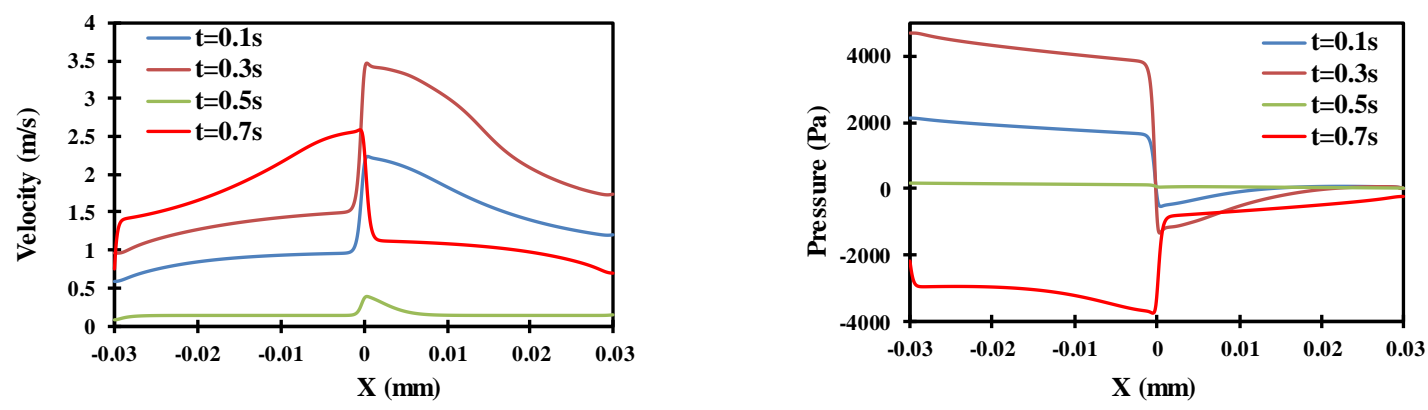

(a)
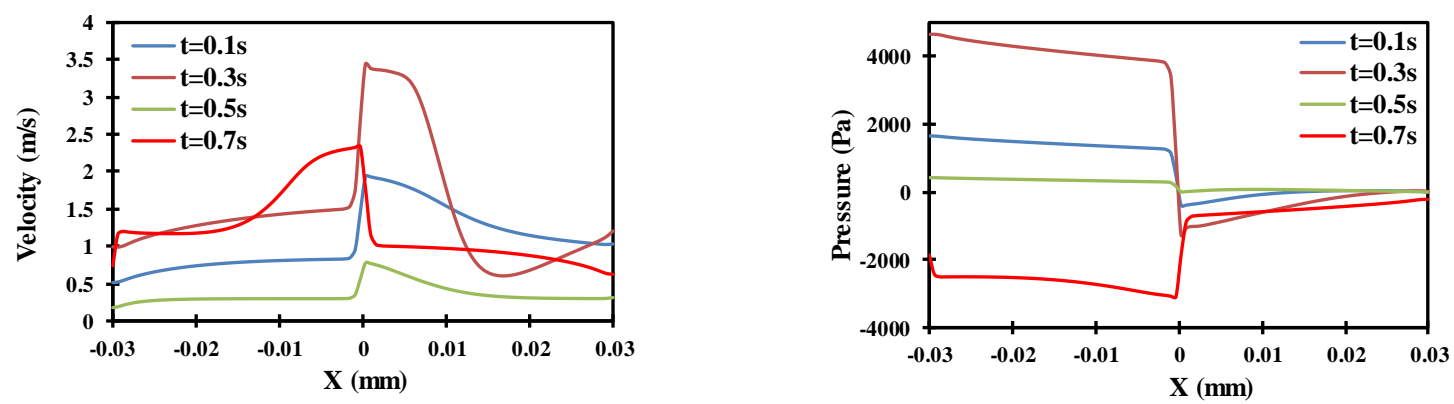

(b)

Figure 15. Velocity and pressure distribution on the central line of the artery for (a) Newtonian and (b) Non-Newtonian fluids equivalent pulsatile flows.
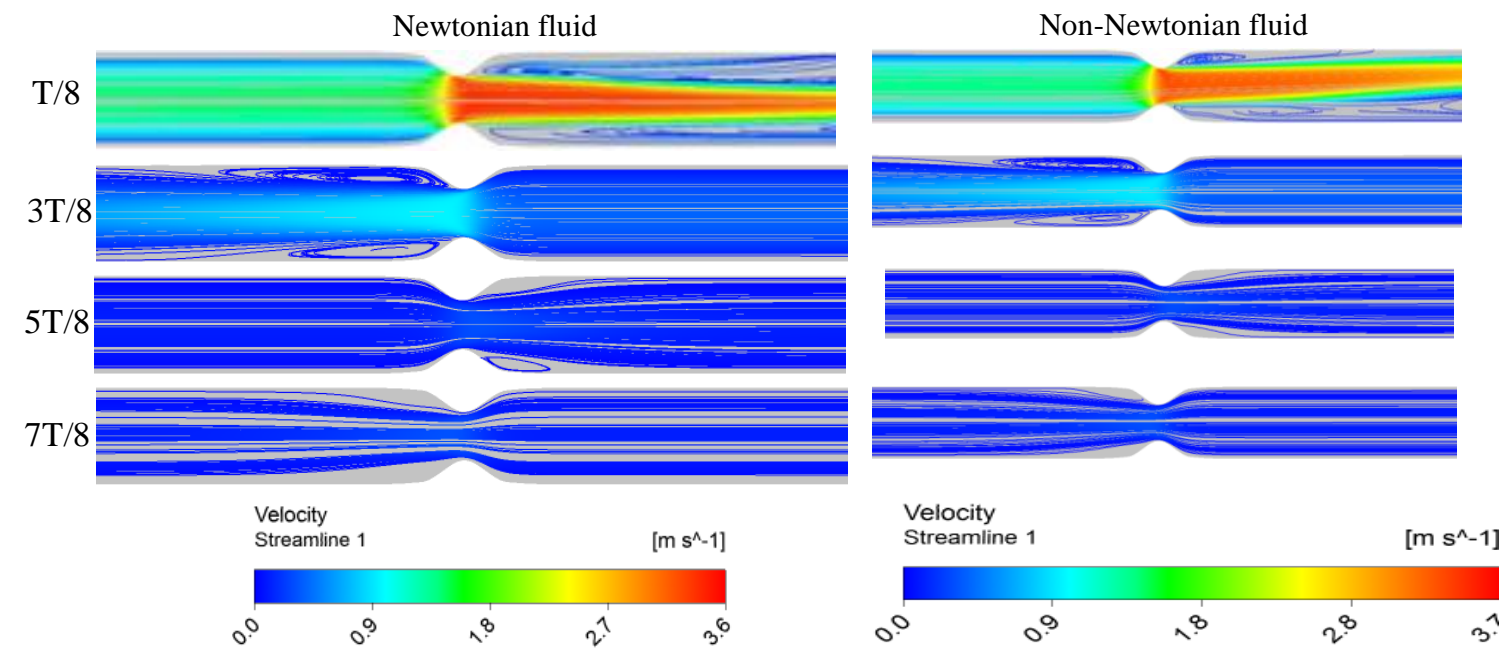

Figure 16. Streamlines at the mid-plane along the flow direction for physiological flows.

- The Newtonian fluid flow produces a large separation regime with vortex for uniform inlet velocity.

- The Newtonian fluid flow gives a higher shear strain rate, whereas the nonNewtonian fluid flow gives a higher wall shear stresses near the bifurcation regime.
- In general, the maximum velocity appears at the middle of the artery. The nonNewtonian fluid pulsatile flow gave a deflated velocity profile as the flow past the stenosis. The Newtonian fluid produces asymmetric velocity distribution as the flow past the stenosis whereas the nonNewtonian fluid gave symmetric flow field under the investigated flow conditions. 

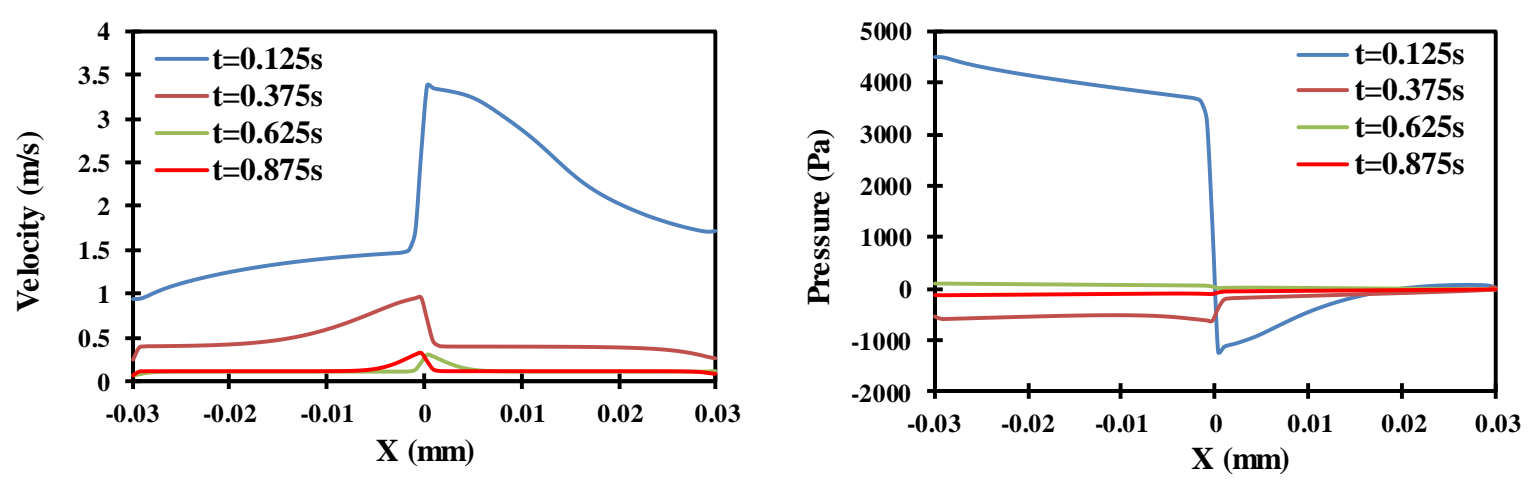

(a)
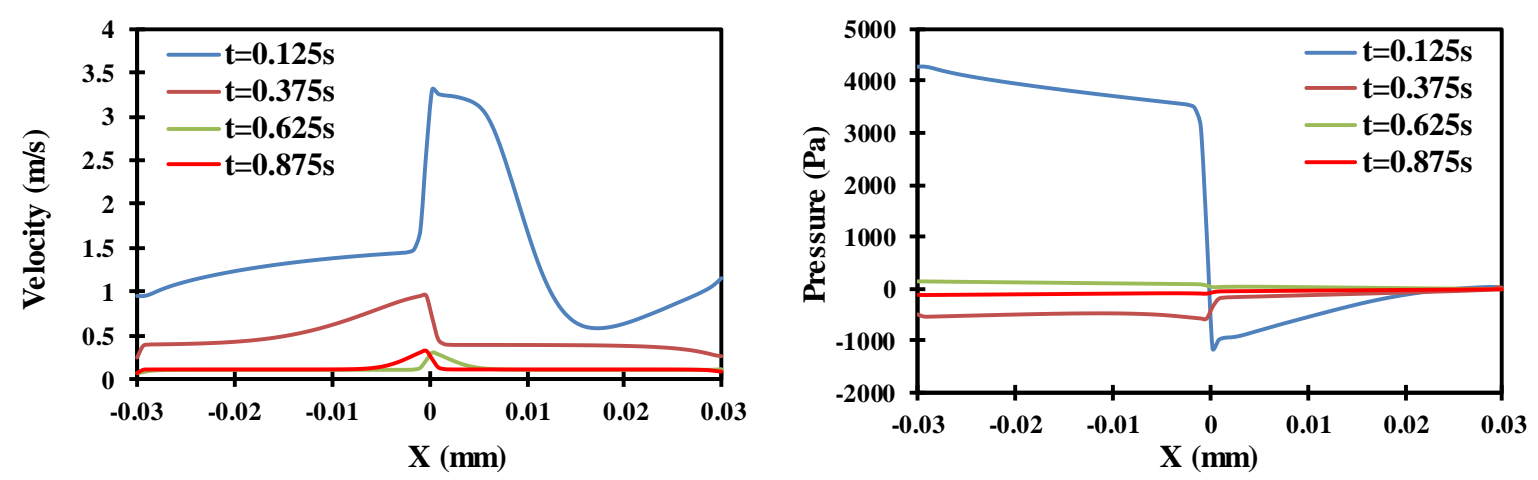

(b)

Figure 17. Velocity and pressure distribution on the mid line of the artery for (a) Newtonian and (b) Non-Newtonian fluids physiological flows.

- The pressure drops sharply near the stenosis and recovers gradually as the flow past the stenosis.

- The vortex and low-pressure zone remains proximal to the stenosis for the equivalent pulsatile flow. The vortex location keeps changing with the forward and backward flows during the pulsating cycle. The physiological flow produces the longest vertical regime among the types of flows investigated in the present study.

\section{Conflict of Interest}

The authors declare no conflicts of interest.

\section{Funding}

The authors acknowledge the support of Sultan Qaboos University through Collaborative Research Program (CL/SQU-UAEU/15/02) for conducting this research.

\section{References}

Ahsaas S, Tiwari S (2016), Numerical Simulation of Blood Flow through Asymmetric and Symmetric Occlusion in Carotid Artery. In: Proceedings of the 3rd International Conference on Fluid Flow, Heat and Mass Transfer (FFHMT'16). Ottawa, Canada 1708.

Ang C, Mazumdar JN (1997), Mathematical Flow through Modelling of ThreeDimensional an Asymmetric Arterial Stenosis. Mathl. Comput. Modelling 25: 19-29.

Ansys CFX (2010), Release 11.0: Ansys CFXSolver Theory Guide. ANSYS.

Bharadvaj BK, Mabon RF, Giddens DP (1982a), Steady flow in a model of the human carotid bifurcation. Part I-Flow visualization. Journal of Biomechanics 15:349362. doi: 10.1016/0021-9290(82)90057-4.

Bharadvaj BK, Mabon RF, Giddens DP (1982b), Steady flow in a model of the human carotid bifurcation. Part II-Laser-Doppler anemometer measurements. Journal of Biomechanics 15:363-378. doi: 10.1016/00219290(82)90058-6. 
Chakravarty S, Mandal PK (1994), Mathematical modelling of blood flow through an overlapping arterial stenosis. Mathematical and Computer Modelling 19:59-70. doi: 10.1016/0895-7177(94)90116-3.

Chiastra C, Gallo D, Tasso P, Iannaccone F, Migliavacca F, Wentzel JJ, Morbiducci U (2017), Healthy and diseased coronary bifurcation geometries influence near-wall and intravascular flow: A computational exploration of the hemodynamic risk. Journal of Biomechanics 58:79-88. doi: 10.1016/j.jbiomech.2017.04.016.

Deplano V, Siouffi M (1999), Experimental and numerical study of pulsatile flows through stenosis: Wall shear stress analysis. Journal of Biomechanics 32:1081-1090. doi: 10.1016/S0021-9290(99)00098-6.

Deshpande MD, Giddens DP, Mabon RF (1976), Steady laminar flow through modelled vascular stenoses. Journal of Biomechanics 9:165-174. doi: 10.1016/0021-9290(76)900014.

Ellahi R, Rahman SU, Nadeem S (2014), Blood flow of Jeffrey fluid in a catherized tapered artery with the suspension of nanoparticles. Physics Letters, Section A: General, Atomic and Solid State Physics 378:2973-2980. doi: 10.1016/j.physleta.2014.08.002.

Gijsen FJH, van de Vosse FN, Janssen JD (1999), The influence of the non-Newtonian properties of blood on the flow in large arteries: steady flow in a carotid bifurcation model. J Biomech 32: 601-608.

Hye MA, Paul MC (2015), A computational study on spiral blood flow in stenosed arteries with and without an upstream curved section. Applied Mathematical Modelling 39:4746-4766. doi: 10.1016/ j.apm.2015.04.019.

Jozwik K, Obidowski D (2008), Geometrical models of vertebral arteries and numerical simulations of the blood flow through them. In: Proceedings of the 3rd Frontiers in Biomedical Devices Conference and Exhibition. June 18-20, Irvine, California, USA. 75-79.

$\mathrm{Ku}$ DN, Giddens DP, Zarins CK, Glagov S (1985), Pulsatile flow and atherosclerosis in the human carotid bifurcation. Arterioscler Thromb Vasc Biol 5:293-302. doi: 10.1161/01.ATV.5.3.293.

Liao W, Lee TS, Low HT (2004), Numerical studies of physiological pulsatile flow through constricted tube. International Journal of Numerical Methods for Heat and
Fluid Flow 14:689-713. doi: 10.1108/09615530410539991.

Long Q, Xu XY, Ramnarine K V., Hoskins P (2001), Numerical investigation of physiologically realistic pulsatile flow through arterial stenosis. Journal of Biomechanics 34:1229-1242. doi: 10.1016/S0021-9290(01)00100-2.

MazHer AHK, Ekaterinaris JA (1988), Numerical simulation of blood flow through arteries. In: Proceedings of the 1988 Fourteenth Annual Northeast Bioengineering Conference. 10-11 March; Durham, NH, USA, USA, 273-276.

Mcdonald DA (1955), The Relation of Pulsatile Pressure To Flow in Arteries. J Physiol I27:533-552. doi: 10.1113 / jphysiol. 1955. sp005275.

Mekheimer KS, El Kot MA (2015), Suspension model for blood flow through catheterized curved artery with time-variant overlapping stenosis. Engineering Science and Technology, an International Journal 18:452-462. doi: 10.1016/j.jestch.2015.03.010.

Nadeem S, Akbar NS, Hendi AA, Hayat T (2011), Power law fluid model for blood flow through a tapered artery with a stenosis. Applied Mathematics and Computation 217:7108-7116. doi: 10.1016/j.amc.2011.01.026.

Perktold K, Rappitsch G (1995), ComputerSimulation of Local Blood-Flow and Vessel Mechanics in a Compliant Carotid-Artery Bifurcation Model. Journal of Biomechanics 28:845-856. doi: 10.1016/0021-9290(95) 95273-8.

Perktold K, Resch M, Peter RO (1991), Threedimensional numerical analysis of pulsatile flow and wall shear stress in the carotid artery bifurcation. Journal of Biomechanics 24:409-420. doi: 10.1016/00219290(91)90029-M.

Razavi A, Shirani E, Sadeghi MR (2011), Numerical simulation of blood pulsatile flow in a stenosed carotid artery using different rheological models. Journal of Biomechanics 44:2021-2030. doi: 10.1016/j.jbiomech.2011.04.023.

Srivastava VP, Rastogi R (2010), Blood flow through a stenosed catheterized artery: Effects of hematocrit and stenosis shape. Computers and Mathematics with Applications 59:1377-1385. doi: 10.1016/j.camwa.2009.12.007.

Tu C, Deville M (1996), Pulsatile flow of NonNewtonian fluids through arterial stenoses. 
Journal of Biomechanics 29:899-908. doi: 10.1016/0021-9290(95)00151-4.

Tu C, Deville M, Dheur L, Vanderschuren L (1992), Finite element simulation of pulsatile flow through arterial stenosis. Journal of Biomechanics 25:1141-1152. doi: 10.1016/0021-9290(92)90070-H.

Van Doormaal JP, Raithby GD (1984), Enhancements of the simple method for predicting incompressible fluid flows. Numerical Heat Transfer 7:147-163. doi: 10.1080/01495728408961817.
Young DF, Tsai FY (1973), Flow characteristics in models of arterial stenosis - I. Steady flow. Journal of biomechanics 6:395-410. doi: 10.1016/0021-9290(73)90099-7.

Zendehbudi GR, Moayeri MS (1999), Comparison of physiological and simple pulsatile flows through stenosed arteries. Journal of Biomechanics 32:959-965. doi: 10.1016/S0021-9290(99)00053-6. 